\title{
Sylvio Ferraz-Mello: Canonical Perturbation Theories. Degenerate Systems and Resonance
}

\author{
Springer, New York 2007
}

\author{
Alessandra Celletti
}

Received: 5 February 2008 / Accepted: 6 February 2008 / Published online: 28 February 2008

(C) Springer Science+Business Media B.V. 2008

The book by Sylvio Ferraz-Mello provides a deep insight in perturbation theories. As is well known, the most impressive astronomical implementations of such theories dates back to the nineteenth century, when they were used to compute approximations of the celestial motions. Among the others, it is worth mentioning the master work of Charles Delaunay, where the motion of the Moon was computed with astonishing precision. Even more striking was the discovery of Neptune by Urban Le Verrier and John Adams, as a result of the computation of the perturbations induced by an unknown planet on the anomalous motion of Uranus. Perturbation theories were the starting point of the seminal work of Henri Poincaré on the three-body dynamics, which led to substantial improvements in Celestial Mechanics and which gave a deep imprint in the theory of Dynamical Systems. At the middle of the twentieth century, the small divisor problem, which in some cases prevents the application of perturbation theories, was overcome by Andrei N. Kolmogorov who proved the survival of invariant tori under perturbation; his result was later extended in different settings by Vladimir Arnold and Jürgen Moser, giving rise to the so-called KAM theory.

All these theories have a common leit-motiv: they can be constructively applied to provide concrete results; in other words, while maintaining the mathematical rigor, perturbation theories may contribute to give meaningful physical applications. This feature is one of the most attractive aspects of "Canonical Perturbation Theories" by Sylvio Ferraz-Mello, where the mathematical presentation of the different facets is always accompanied by a number of case studies and examples. Such peculiarity makes the book unique and allows the reader to deeply understand the theory through many concrete implementations.

It is worth mentioning that the models of Celestial Mechanics often present troubles with respect to a direct implementation of classical perturbation theory: for example, most dynamical models are degenerate or move in a resonance. All these cases are exhaustively investigated in the book through a proper development of the theory and a suitable presentation of many examples to appreciate the theory in concrete applications.

\footnotetext{
A. Celletti $(\varangle)$

Dipartimento Di Matematica, Università di Roma Tor Vergata,

Via Della Ricerca Scientifica, 1, Roma 00133, Italy

e-mail: celletti@axp.mat.uniroma2.it
} 
The book is structured into ten chapters and four appendixes. Chapter 1 deals with the foundations of Hamiltonian mechanics with particular reference to Hamilton-Jacobi theory, beside the description of Hamilton's principle, the introduction of canonical transformations and the discussion of Liouville's theorem on integrable systems. The introduction of actionangle variables is presented in Chapt. 2, where separable systems are also described and the example of Kepler's motion is discussed. Classical perturbation theory using the Jacobi's generating function is presented in Chapt. 3; here an exhaustive treatment of the theories for different Hamiltonian systems is presented: from the non-degenerate Poincare theory to the Zeipel-Brouwer approach for degenerate systems. Small divisors, resonances and secular motions are also discussed; the chapter is completed by a discussion on Kolmogorov's theorem and on the frequency relocation. Chapter 4 is devoted to resonances: the chapter opens with the discussion of Delaunay's lunar theory and proceeds with the presentation of the paradigmatic model provided by the Garfinkel's Ideal Resonance Problem for which action-angle variables are provided in all typical dynamical regimes, namely circulation and libration; the method of successive eliminations of harmonics is presented and extensively implemented on a specific Hamiltonian system. Lie series are introduced in Chapt. 5. First it is shown that Lie mappings are canonical and can be used for perturbation theories, which is the subject of Chapt. 6; here a non-degenerate perturbation theory using Lie series is presented; a comparison with Poincaré theory is performed and widely implemented on two concrete examples. A relevant part of this chapter concerns Hori's general theory including the introduction of the Hori kernel, the appearance of small divisors and the construction of Hori's formal first integrals. Many interesting problems of Celestial Mechanics present singularities of the action variables. It is therefore important to introduce non-singular variables as discussed in Chapt. 7; in particular, Poincaré non-singular canonical variables are presented and a Lie series perturbation theory in non-singular variables is discussed using Hori theory. Chapter 8 deals with the development of Lie series for resonant systems. Bohlin's problem is introduced to describe the case of a Hamiltonian system including simultaneously resonant and degenerate angles. The solution of this problem is constructed using a Lie series approach. The case of one resonance near a singularity is discussed in Chapt. 9; first, the study of a Hamiltonian with one degree of freedom is discussed. Next, a more dimensional example with a single resonance is worked out; the chapter closes with the treatment of the integrability of a Hamiltonian function through Sessin transformation. Chapter 10 deals with nonlinear oscillators; first, a quasi-harmonic Hamiltonian system is discussed and a formal solution through Hori theory is presented. After exploiting the resonance conditions, the Birkhoff normalization is developed using Lie series. The dynamics of the triangular Lagrangian points in the framework of the restricted three-body problem is provided and a discussion is devoted to the 2:1 and 3:1 internal resonances. The quasi-harmonic Hénon-Heiles Hamiltonian with one commensurability is presented. After discussing the case of multiple commensurabilities, the chapter is concluded with the discussion of a parametrically excited system, like a linear oscillator with a time-dependent frequency.

A relevant role in this book is played by four seminal appendixes. The first one exploits in details Bohlin's theory through a perturbative approach and discusses the singularity of the homological equation in the case of librations. The second appendix deals with the simple pendulum providing action-angle variables in the circulation and libration regimes, as well as a discussion of its dynamics from the small oscillations to the motion close to the separatrix, whose study leads to the introduction of the paradigmatic standard map. The third appendix exploits the Andoyer Hamiltonian with the periodic part depending on the square root of the action; in order to study the associated phase portrait, the singular points are determined and the extent of the librational zone is computed; finally the equilibrium points and 
the small amplitude librations are explored. The last appendix is devoted to the study of the Andoyer Hamiltonians with the periodic part of higher order with respect to that of the previous appendix. Again, phase portraits and librational zones are studied in detail.

This promenade along the different chapters of the book might give an idea of how complete and exhaustive is the variety of topics presented in connection to several kinds of perturbation theories. Thanks to the large scientific expertise of the author, the book is recommended for both teaching and research purposes: the didactical and self-contained aspects address the book to advanced undergraduate students; the researchers in Dynamical Systems and Celestial Mechanics will certainly enjoy the excellent and deep presentation of perturbation theories. 